\title{
Reconfigurable Optical Networks: a Cross Layer Approach
}

Reza Roshani, Paolo Monti, Marco Tacca, and Andrea Fumagalli

OpNeAR Lab, Erik Jonsson School of Engineering and Computer Science

The University of Texas at Dallas, Richardson, TX 75080, USA

\{rxr058100, paolo, mtacca, andreaf \}@utdallas.edu

\begin{abstract}
This paper presents the Pruning with Memory (PWM) algorithm, which computes a cross layer optimal reconfiguration sequence in reconfigurable IP/MPLS over optical networks.
\end{abstract}

\section{Introduction}

Reconfigurable optical networks [1] may yield tangible advantages, in terms of network utilization, when the offered traffic at the upper layers (e.g., IP/MPLS) fluctuates over time. Reconfiguration of the optical layer requires intermediate steps, as it is possible to set up/tear down only a limited number of lightpaths concurrently. The work in [3] presents an algorithm to identify the minimum number of lightpath moves to transition from one configuration step to the next, but no sequence is provided, i.e., all sequences are equivalent. However, in an IP/MPLS over optical network, different sequences of intermediate steps impact differently the IP/MPLS layer. During reconfiguration, LSP's must adapt to the underlying logical topology changes, i.e., LSP's traffic may be rerouted, buffered or, in some cases, (partially or totally) disrupted. Identifying the optimal sequence of intermediate steps and LSP's rerouting to transition the network from one state to the next, becomes a cross layer key objective in order to determine the success of reconfigurable optical networks.

This paper presents the Pruning with Memory (PWM) algorithm, a cross layer solution that identifies the optimal reconfiguration sequence while minimizing disruption at the IP/MPLS layer. The set of possible reconfiguration sequences $S_{R}$ with the best number of moves is obtained from the permutations of the elements in the set identified in [2]. PWM identifies the optimal reconfiguration sequence by intelligently pruning set $S_{R}$ based on two factors: (a) the minimization of metrics of interest, e.g., average hop count and loss at the current reconfiguration step, and (b) the effect of previous reconfiguration steps.

The proposed PWM algorithm is then compared to the MAPF algorithm [2]. The MAPF algorithm is a greedy algorithm that determines the optimal reconfiguration sequence irrespective of the loss of traffic at the IP/MPLS layer. Numerical results show that the presented approach reports gains, in terms of bandwidth loss, as high as $50 \%$.

\section{Network Model}

Consider a network where each node comprises both an IP/MPLS router and an optical cross-connect
(OXC). OXC's are used to establish lightpaths, i.e., provide a virtual topology to the routers. The following assumptions are made: (i) the number of wavelengths is unbounded, (ii) traffic is symmetric, i.e, for every LSP from node $s$ to node $d$ there is another LSP with the same bandwidth requirement routed in the reverse direction, (iii) LSP's may be split and carried over multiple paths on the virtual topology. Two LSP traffic matrices are considered, $\Lambda_{\text {in }}$ and $\Lambda_{\text {fin }}$. The initial virtual topology $G_{i n}\left(N, A_{i n}\right)$ is the virtual topology resulting from the traffic matrix $\Lambda_{\text {in }}$, while the final virtual topology $G_{\text {fin }}\left(N, A_{\text {fin }}\right)$ is the virtual topology resulting from the traffic matrix $\Lambda_{\text {fin }} . N$ is the set of routers in the network, while $A_{\text {in }}$ and $A_{\text {fin }}$ are the set of lightpaths connecting the routers in the initial and final virtual topology, respectively. $\mathrm{G}_{\text {in }}\left(\mathrm{N}, \mathrm{A}_{\text {in }}\right)$ and $\mathrm{G}_{\text {fin }}\left(\mathrm{N}, \mathrm{A}_{\text {fin }}\right)$ are computed using the procedure presented in [1].

Let $S=\left\{S t_{0}, S t_{1}, \ldots, S t_{M}\right\}$ be a reconfiguration sequence able to transform $G_{\text {in }}\left(N, A_{\text {in }}\right)$ into $G_{\text {fin }}\left(N, A_{\text {fin }}\right)$ in $M$ reconfiguration steps. Let $G_{0}=G_{i n}\left(N, A_{\text {in }}\right)$ and $G_{M}=$ $\mathrm{G}_{\text {fin }}\left(N, A f_{\text {in }}\right)$. During a reconfiguration step $\left(S t_{n}\right)$, virtual topology $G_{n-1}$ is transformed into virtual topology $G_{n}$ by tearing down and/or setting up a limited number of lightpaths. The execution of each step requires a time $T$. The execution of the reconfiguration sequence up to step $S t_{n}$ requires a time $T_{n}=n T$. Routing of LSP's in $G_{n}$ is computed using the model presented in [1] modified to minimize the LSP traffic loss (if any) as follows. First a cost function is introduced. The cost function is defined as:

$$
\text { Loss }=\min B L, \quad B L=\sum_{i, j}\left(\lambda_{(i, j)}^{(n)}-k_{(i, j)}^{(n)} \cdot \lambda_{(i, j)}^{(n)}\right)
$$

where the bandwidth loss $(\mathrm{BL})$ measures the total traffic disruption in the network due to lack of resources. $\lambda_{(i, j)}^{(n)}$ is the bandwidth requirement of an LSP from node $i$ and node $j$ at time instance $(n)$, and $k_{(i, j)}^{(n)} \cdot \lambda_{(i, j)}^{(n)} \quad$ (with $\left.k_{(i, j)}^{(n)} \leq 1\right)$ is the portion of the bandwidth of the LSP that is actually routed. In all the constraints of the flow model defined in [1] $\lambda_{(i, j)}^{(n)}$ is replaced by $k_{(i, j)}^{(n)} \cdot \lambda_{(i, j)}^{(n)}$. 


\section{The Pruning with Memory (PWM) Algorithm}

$G_{\text {in }}\left(N, A_{\text {in }}\right)$ and $G_{\text {fin }}\left(N, A f_{\text {in }}\right)$ are computed using the method explained in the previous section. The set of all possible reconfiguration sequences $\left(S_{R}\right)$ able to transform $G_{\text {in }}$ into $G_{\text {fin }}$ with the minimum number of reconfiguration steps $(\mathrm{M})$ is precomputed using the algorithm presented in [3].

The objective of the PWM algorithm is to find one reconfiguration sequence $S_{\min }$ in set $S_{R}$ which is able to minimize the value of the bandwidth loss (avgB $L_{\text {min }}$ ). $S_{\min }$ is found by pruning $S_{R}$ as explained by the pseudo code in Figure 1. Notice how the PWM algorithm, using the margin parameter, takes into account the effect of previous reconfiguration steps.

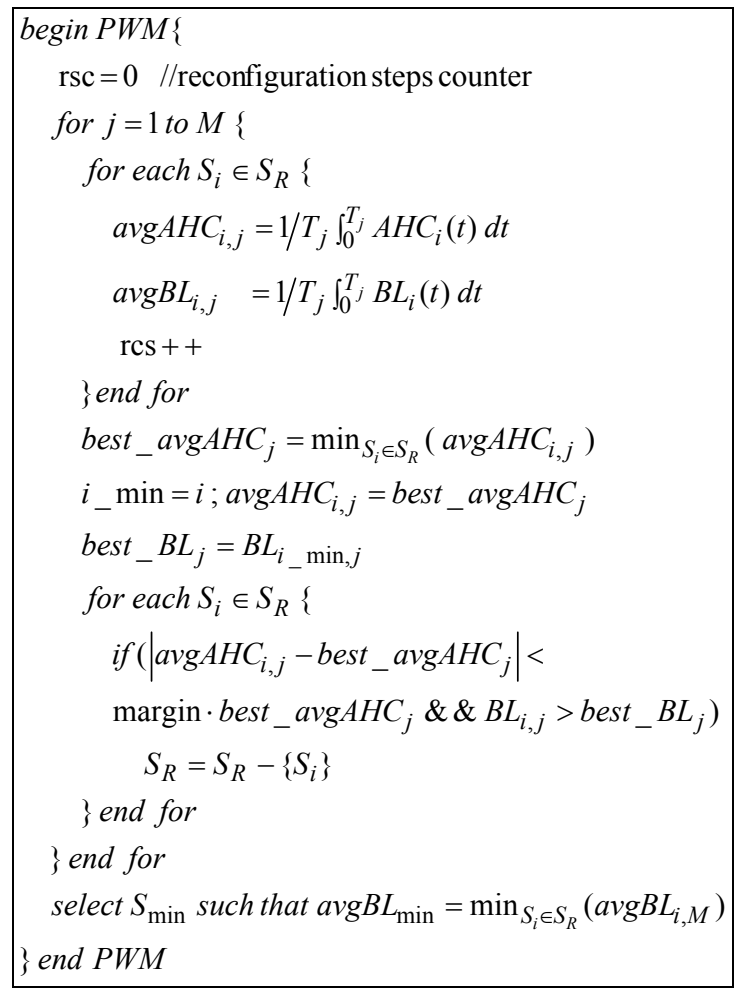

Figure 1: Pseudo code of the PWM algorithm

\section{Simulation Results}

This section compares the PWM and the MAPF algorithm in terms of $a v g B L_{\min }$. Numerical results are obtained for a network of 10 nodes. Each node has three transmitters and three receivers. The lightpath capacity is $C=10 \mathrm{Gbps}$. Transmission impairments and traffic fluctuations are modelled using set $V_{i}$ and parameters $f_{p}$ and $f_{t}$ as explained in [1]. $\Lambda_{\text {in }}$ and $\Lambda_{\text {fin }}$ are assigned the same values used in [1]. The margin parameter is set equal to 1 . Presented results are averages of 100 experiments. For each experiment $\Lambda_{\text {in }}, \Lambda_{\text {fin }}, f_{p}$ and $f_{t}$ are left unchanged. Only the neighboring set $V_{i}$ is randomly generated for each node $i$.

Figure 2 shows the value of $a v g B L_{\min }$ as a function of the LSP's traffic fluctuations. Results confirm the earlier claim that considering the effect of the previous configurations steps has a beneficial effect on the traffic disruption at upper layers. The additional complexity required by the PWM algorithm is shown in Figure 3, where the value of the reconfiguration steps counter (rsc) (Figure 1) is presented as a function of the LSP's traffic fluctuations.

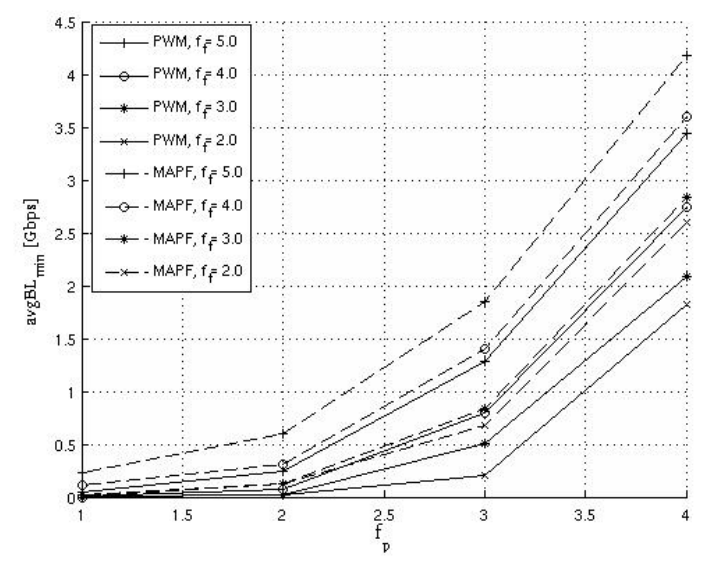

Figure 2: average bandwidth loss (avgBLmin) as a function of the LSP's traffic fluctuations

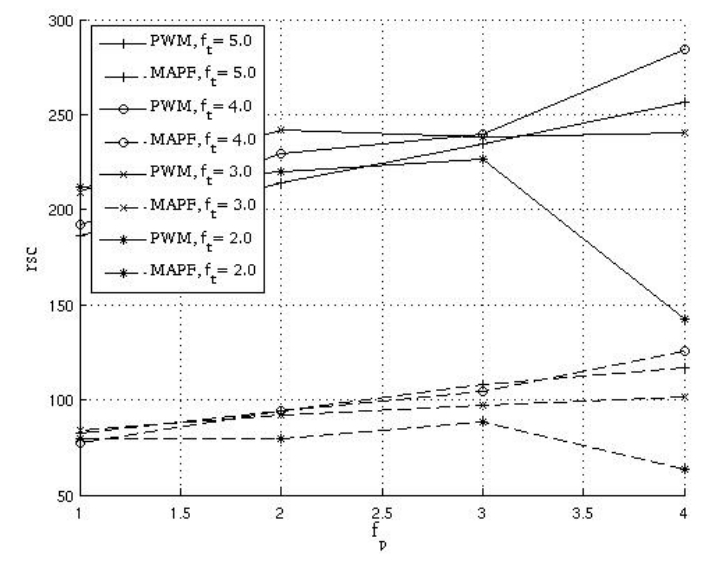

Figure 3: reconfiguration steps counter (rsc) as a function of the LPS's traffic fluctuations

\section{Conclusions}

This paper presented the PWM algorithm, a cross layer solution that reconfigures the optical layer while minimizing traffic disruption at the IP/MPLS layer. Numerical results show that the presented approach is able to reduce the traffic disruptions by up to $50 \%$.

\section{References}

1. R. Roshani, et al. OFC2008, OTuA2

2. Y. Zhang, et al. Journal of Lightwave Technology, Volume 23 (2005), 2854-2867

3. J. F. P. Labourette, et al. IEEE Trans. Commun., Volume 42, no.10 (1994), 2822-2832 